\title{
Scaling Laws for One-dimensional Ad Hoc Wireless Networks
}

\author{
Olivier Lévêque, Emmanuel Preissmann \\ Ecole Polytechnique Fédérale de Lausanne, Switzerland
}

December 15, 2004

\begin{abstract}
We obtain a precise information theoretic upper bound on the rate per communication pair in a one-dimensional ad hoc wireless network. The key ingredient of our result is a uniform upper bound on the determinant of the Cauchy matrix.
\end{abstract}

\section{Introduction}

The study of the capacity of ad hoc wireless networks goes back to the seminal paper of P. Gupta and P. R. Kumar [3], in which they prove, under some realistic assumptions regarding state of the art wireless communications, that the transport capacity of planar ad hoc networks grows asymptotically at most like the square root of the number of users in the network. One still misses a confirmation of this result from an information theoretic point of view (i.e., without any assumption on the way communications are established in the network). Some attempts have been performed recently (see for instance $[4,5,6]$ ), all leading to partial answers.

The argument of Gupta and Kumar can be easily translated to one-dimensional networks and leads to the conlusion that in this case, the transport capacity of the network grows asymptotically at most like the number of users. Even though the analysis is (much) simpler in the one-dimensional case, no complete information theoretic confirmation of this result has been given so far.

In [5], P. R. Kumar and L.-L. Xie consider an arbitrary one-dimensional network composed of $n$ users separated by a minimum distance $d>0$ and show that the transport capacity of such networks does not grow faster than $n$, provided that the attenuation function of the transmitted signals over distance is given by

$$
g(r)=\frac{e^{-\beta r / 2}}{r^{\alpha / 2}}
$$

where either $\beta>0$ or $\alpha>4$ (note that $g$ describes the decay of the amplitude of the electric field and not that of the power).

In the particular scenario where order $n$ pairs chosen at random wish to establish communication, the above result implies that the maximum achievable rate $R$ per communication pair decreases like $\frac{1}{n}$ as $n$ gets large, since order $n$ communications need to be established over distances of order $n$ on average (recall that the network is assumed to be one-dimensional and that users are separated by a minimum distance $d$ ).

In the case $\beta=0$, the above assumption that $\alpha>4$ is quite unrealistic regarding wireless communications. In [6], O. Lévêque and E. Telatar consider uniformly distributed one-dimensional 
networks (with a constant density of users) and show that in the above mentioned scenario, if $\alpha>1$, then

$$
R \leq K \frac{\log n}{n^{1-\frac{1}{\alpha}}}
$$

so that $R$ tends to zero as $n$ gets large. However, this upper bound is as not tight as the $\frac{1}{n}$ behaviour obtained under a weaker assumption on $\alpha$ in [5]. In the following, we consider an arbitrary one-dimensional network with users separated by a minimum distance $d>0$ and show (see theorem 3.1) that if $2 \leq \alpha \leq 4$, then the following upper bound holds true:

$$
\forall \varepsilon>0, \quad \exists K>0 \quad \text { such that } \quad R \leq K \frac{(\log n)^{3+\varepsilon}}{n},
$$

which is tighter than (1), especially for small $\alpha$. With this respect, our result below closes the information theoretic gap left open in [5] concerning one-dimensional ad hoc networks.

Note finally that a result similar in spirit has been obtained by A. Jovicic et al. in [4]: for one-dimensional networks and under the slightly different propagation model

$$
\tilde{g}(r)=\frac{e^{i \phi}}{(1+r)^{\alpha / 2}}
$$

where $\phi$ is a random phase, they prove that the transport capacity does not grow faster than $n$, provided that $\alpha>3$ and that the users have a perfect knowledge of the phases, or provided that $\alpha>2$ but that users have no information about the phases.

\section{Our Approach}

We follow here the lines of [6], specializing the model to arbitrary one-dimensional networks.

We consider a network of $n$ users (with $n$ even for simplicity) arbitrarily placed on the real line, but separated by a minimum distance $d>0$. Among these $n$ users, we choose $n / 2$ users at random and assume that each of these users wishes to establish communication with a correspondent chosen at random in the other group of $n / 2$ users (without any consideration on their respective locations). We assume that there is no fixed infrastructure that helps relaying communications, but we also assume no restriction on the kind of help the users can give to each other; in particular, any user may act as a relay for the communicating pairs, but we may also imagine more sophisticated group communications and interference cancellation strategies. We further assume that in order to establish communication, each user has a device of power $P$. The attenuation of the transmitted signals over distance is governed by the function $g(r)$ given by $g(r)=\frac{1}{r^{\alpha / 2}}$ with $2 \leq \alpha \leq 4$. For notational convenience, let us define the coefficient $\delta=\alpha / 2$ (corresponding to the coefficient $\delta$ defined in [5]).

We divide the network into two parts, so that there are exactly $n / 2$ users on each side, and place the origin at the middle point between the two most "central" users. There are therefore $n / 2$ users located left to the origin; statistically, half of these are transmitters and half of these transmitters wish to establish communication with a receiver located right to the origin. In total, there are therefore about $n / 8$ communications which need to be carried over the origin from left to right, and deviations from this idealized situation are of order much smaller than $n$ with high probability.

Let $R$ be the the maximum achievable rate per communication pair in the network. In order to obtain an upper bound on $R$, we first assume that only the above $n / 8+o(n)$ communications 
need to be established. We then introduce $n$ additional "mirror" user that help relaying communications (where the mirror location of $x \in \mathbb{R}$ is $\tilde{x}=-x$ ). There are now $n$ users on each side of the origin whose positions are denoted respectively by

$$
x_{1}, \ldots, x_{n} \text { and } y_{1}, \ldots, y_{n}, \quad \text { with } y_{i}=-x_{i} .
$$

Without any restriction of generality, we may order the points so that $x_{1} \leq \ldots \leq x_{n}$. By the constraint imposed on the minimum distance, we obtain that $x_{1} \geq \frac{d}{2}$ and that $x_{i} \geq(i-1) \frac{d}{2}$ for all $i \in\{2, \ldots, n\}$.

Using the classical cut-set bound of [2, Thm 14.10.1] and following the argument of [6], we obtain that

$$
R \leq \frac{C_{n}}{n / 8+o(n)},
$$

where $C_{n}$ is the capacity of the vector channel given by

$$
Y_{j}=\sum_{i=1}^{n} G_{i j}^{(\delta)} X_{i}+Z_{j}, \quad j=1, \ldots, n
$$

with

$$
G_{i j}^{(\delta)}=\frac{1}{\left|x_{i}-y_{j}\right|^{\delta}}=\frac{1}{\left(x_{i}+x_{j}\right)^{\delta}}
$$

and $Z=\left(Z_{1}, \ldots, Z_{n}\right)$ is a vector of independent circularly symmetric complex Gaussian random variables with unit variance. Under the power constraint

$$
\sum_{i=1}^{n} \mathbb{E}\left(\left|X_{i}\right|^{2}\right) \leq n P
$$

the capacity of the above channel is given by

$$
C_{n}=\max _{P_{k} \geq 0: \sum_{k=1}^{n} P_{k} \leq n P} \sum_{k=1}^{n} \log \left(1+P_{k} \lambda_{k}^{2}\right),
$$

where $\lambda_{k}$ are the eigenvalues of the symmetric matrix $G^{(\delta)}$. Noting that $P_{k} \leq n P$ for each $k$ and that the $\lambda_{k}$ are non-negative (see [6]), we further obtain that

$$
C_{n} \leq \sum_{k=1}^{n} \log \left(1+n P \lambda_{k}^{2}\right) \leq 2 \sum_{k=1}^{n} \log \left(1+\sqrt{n P} \lambda_{k}\right)=2 \log \operatorname{det}\left(I+\sqrt{n P} G^{(\delta)}\right) .
$$

\section{Main Result}

Theorem 3.1. Let $1 \leq \delta \leq 2$ (or equivalently, $2 \leq \alpha \leq 4)$. For all $\varepsilon>0$, there exists a constant $K>0$ (independent of $n$ and $\delta$ ) such that the capacity $C_{n}$ is bounded above by

$$
C_{n} \leq K(\log n)^{3+\varepsilon}, \quad \forall n \geq 1,
$$

so this estimate combined with (3) implies the upper bound (2) on the maximum achievable rate $R$ per communication pair in the network. 
Remark 3.2. Looking into the details of the proof below shows that the constant $K$ is actually proportional to $\log \left(\frac{1}{d}\right)$, so that if we consider that the minimum distance $d$ between users is of order $\frac{1}{n^{\eta}}$ with $\eta>0$, then

$$
C_{n} \leq \tilde{K}(\log n)^{4+\varepsilon},
$$

In particular, if we consider $n$ users independently and uniformly distributed on the line segment $[-n, n]$ (resp. $[-1,1]$ ), then typically $d$ is of order $\frac{1}{n}$ (resp. $\left.\frac{1}{n^{2}}\right)$, so the above argument applies.

\section{Scheme of the proof of theorem 3.1.}

The proof starts with the following identity, valid for any $n \times n$ matrix $A$ :

$$
\operatorname{det}(I+A)=\sum_{J \subset\{1, \ldots, n\}} \operatorname{det}(A(J)), \quad \text { where } A(J)=\left(a_{i j}\right)_{i, j \in J} .
$$

The capacity $C_{n}$ can therefore be expressed in terms of determinants of the form

$$
D_{\delta}\left(\mathbf{x}_{J}\right)=\operatorname{det}\left(\left(\frac{1}{\left(x_{i}+x_{j}\right)^{\delta}}\right)_{i, j \in J}\right), \quad \text { where } \mathbf{x}_{J}=\left(x_{i}\right)_{i \in J} .
$$

The first step of the proof consists in showing that there exists a constant $K>0$ independent of $n$ such that for all $1 \leq \delta \leq 2$,

$$
D_{\delta}\left(\mathbf{x}_{J}\right) \leq m K^{m} D_{1}\left(\mathbf{x}_{J}\right), \quad \text { where } m=|J|,
$$

so that we can concentrate on the case $\delta=1$, for which we have the following nice analytic expression, due to Cauchy (see for instance [7, p. 202]):

$$
D_{1}\left(\mathbf{x}_{J}\right)=\left(\prod_{\substack{i, j \in J \\ i<j}}\left(x_{j}-x_{i}\right)^{2}\right) /\left(\prod_{i, j \in J}\left(x_{i}+x_{j}\right)\right) .
$$

In a second step, we perform a detailed study of the configuration $\mathbf{x}_{J}$ maximizing $D_{1}\left(\mathbf{x}_{J}\right)$ and show that there exist $K_{1}, K_{2}>0$ such that

$$
D_{1}\left(\mathbf{x}_{J}\right) \leq K_{1}^{m} \exp \left(-K_{2} m^{3 / 2}\right) .
$$

Finally, using estimates (8) and (10) together with formula (6) leads to the conclusion.

\section{Proof of theorem 3.1: first step.}

Lemma 3.3. Let $\delta>0$ and $J \subset\{1, \ldots, n\}$. The determinant defined in (7) satisfies the following identity:

$$
D_{\delta}\left(\mathbf{x}_{J}\right)=\frac{1}{m ! \Gamma(\delta)^{m}}\left(\prod_{i \in J} \int_{\mathbb{R}_{+}} d t_{i} t_{i}^{\delta-1}\right) \operatorname{det}\left(\left(e^{-t_{i} x_{j}}\right)_{i, j \in J}\right)^{2},
$$

where $m=|J|$ and $\Gamma$ is the Euler Gamma function.

Proof. Let us first recall that

$$
\frac{1}{\left(x_{i}+x_{j}\right)^{\delta}}=\frac{1}{\Gamma(\delta)} \int_{\mathbb{R}_{+}} d t t^{\delta-1} e^{-t\left(x_{i}+x_{j}\right)} .
$$


For a matrix $A(J)=\left(a_{i j}\right)_{i, j \in J}$, the following formula holds:

$$
\operatorname{det}(A(J))=\sum_{\sigma \in \mathcal{S}(J)} \varepsilon(\sigma) \prod_{i \in J} a_{i, \sigma(i)}=\frac{1}{m !} \sum_{\sigma, \tau \in \mathcal{S}(J)} \varepsilon(\sigma) \varepsilon(\tau) \prod_{i \in J} a_{\sigma(i), \tau(i)},
$$

where $\mathcal{S}(J)$ is the set of permutations of $J$ and $\varepsilon(\sigma)$ is the signature of the permutation $\sigma$. Therefore,

$$
\begin{aligned}
D_{\delta}\left(\mathbf{x}_{J}\right) & =\frac{1}{m ! \Gamma(\delta)^{m}} \sum_{\sigma, \tau \in \mathcal{S}(J)} \varepsilon(\sigma) \varepsilon(\tau) \prod_{i \in J}\left(\int_{\mathbb{R}_{+}} d t_{i} t_{i}^{\delta-1} e^{-t_{i} x_{\sigma(i)}-t_{i} x_{\tau(i)}}\right) \\
& =\frac{1}{m ! \Gamma(\delta)^{m}}\left(\prod_{i \in J} \int_{\mathbb{R}_{+}} d t_{i} t_{i}^{\delta-1}\right)\left(\sum_{\sigma \in \mathcal{S}(J)} \varepsilon(\sigma) \prod_{i \in J} e^{-t_{i} x_{\sigma(i)}}\right)^{2} \\
& =\frac{1}{m ! \Gamma(\delta)^{m}}\left(\prod_{i \in J} \int_{\mathbb{R}_{+}} d t_{i} t_{i}^{\delta-1}\right) \operatorname{det}\left(\left(e^{-t_{i} x_{j}}\right)_{i, j \in J}\right)^{2} .
\end{aligned}
$$

Lemma 3.4. Let $f: \mathbb{R}_{+} \rightarrow \mathbb{R}$ be a measurable function such that both $f(t)^{2}$ and $t f(t)^{2}$ are integrable on $\mathbb{R}_{+}$. Then for all $1 \leq \delta \leq 2$,

$$
\int_{\mathbb{R}_{+}} d t t^{\delta-1} f(t)^{2} \leq\left(\int_{\mathbb{R}_{+}} d t t f(t)^{2}\right)^{\delta-1}\left(\int_{\mathbb{R}_{+}} d t f(t)^{2}\right)^{2-\delta}
$$

Proof. Let us write $t^{\delta-1} f(t)^{2}=u(t) v(t)$, where $u(t)=\left(t f(t)^{2}\right)^{\delta-1}$ and $v(t)=f(t)^{2(2-\delta)}$. Using Hölder's inequality with $p=\frac{1}{\delta-1} \in\left[1, \infty\left[\right.\right.$ and $q=\frac{1}{2-\delta} \in\left[1, \infty\left[\right.\right.$ (so that $\frac{1}{p}+\frac{1}{q}=1$ ), we obtain

$$
\begin{aligned}
\int_{\mathbb{R}_{+}} d t u(t) v(t) & \leq\left(\int_{\mathbb{R}_{+}} d t u(t)^{\frac{1}{\delta-1}}\right)^{\delta-1}\left(\int_{\mathbb{R}_{+}} d t v(t)^{\frac{1}{2-\delta}}\right)^{2-\delta} \\
& =\left(\int_{\mathbb{R}_{+}} d t t f(t)^{2}\right)^{\delta-1}\left(\int_{\mathbb{R}_{+}} d t f(t)^{2}\right)^{2-\delta} .
\end{aligned}
$$

Lemma 3.5. For all $J \subset\{1, \ldots, n\}$ and $1 \leq \delta \leq 2$,

$$
D_{\delta}\left(\mathbf{x}_{J}\right) \leq \frac{1}{\Gamma(\delta)^{m}} D_{2}\left(\mathbf{x}_{J}\right)^{\delta-1} D_{1}\left(\mathbf{x}_{J}\right)^{2-\delta}
$$

where $m=|J|$.

Proof. Let $\mathbf{t}_{J}=\left(t_{i}\right)_{i \in J}$. A multidimensional version of lemma 3.4 shows that for a measurable function $f: \mathbb{R}_{+}^{m} \rightarrow \mathbb{R}$,

$$
\left(\left(\prod_{i \in J} \int_{\mathbb{R}_{+}} d t_{i} t_{i}^{\delta-1}\right) f\left(\mathbf{t}_{J}\right)^{2}\right) \leq\left(\left(\prod_{i \in J} \int_{\mathbb{R}_{+}} d t_{i} t_{i}\right) f\left(\mathbf{t}_{J}\right)^{2}\right)^{\delta-1}\left(\left(\prod_{i \in J} \int_{\mathbb{R}_{+}} d t_{i}\right) f\left(\mathbf{t}_{J}\right)^{2}\right)^{2-\delta}
$$


provided that both integrals on the right-hand side are finite. From this and lemma 3.3, we deduce that

$$
\begin{aligned}
D_{\delta}\left(\mathbf{x}_{J}\right)= & \frac{1}{m ! \Gamma(\delta)^{m}}\left(\prod_{i \in J} \int_{\mathbb{R}} d t_{i} t_{i}^{\delta-1}\right) \operatorname{det}\left(\left(e^{-t_{i} x_{j}}\right)_{i, j \in J}\right)^{2} \\
\leq & \frac{1}{m ! \Gamma(\delta)^{m}}\left(\left(\prod_{i \in J} \int_{\mathbb{R}} d t_{i} t_{i}\right) \operatorname{det}\left(\left(e^{-t_{i} x_{j}}\right)_{i, j \in J}\right)^{2}\right)^{\delta-1} \\
& \cdot\left(\left(\prod_{i \in J} \int_{\mathbb{R}} d t_{i}\right) \operatorname{det}\left(\left(e^{-t_{i} x_{j}}\right)_{i, j \in J}\right)^{2}\right)^{2-\delta} \\
= & \frac{1}{m ! \Gamma(\delta)^{m}}\left(m ! \Gamma(2)^{m} D_{2}\left(\mathbf{x}_{J}\right)\right)^{\delta-1}\left(m ! \Gamma(1)^{m} D_{1}\left(\mathbf{x}_{J}\right)\right)^{2-\delta} \\
= & \frac{1}{\Gamma(\delta)^{m}} D_{2}\left(\mathbf{x}_{J}\right)^{\delta-1} D_{1}\left(\mathbf{x}_{J}\right)^{2-\delta},
\end{aligned}
$$

since $\Gamma(2)=\Gamma(1)=1$.

Let us recall the definition of the permanent of a $m \times m$ matrix $A(J)=\left(a_{i j}\right)_{i, j \in J}$ :

$$
\operatorname{perm}(A(J))=\sum_{\sigma \in \mathcal{S}(J)} \prod_{i \in J} a_{i, \sigma(i)} .
$$

We also define for $\delta>0$ and $J \subset\{1, \ldots, n\}$ :

$$
P_{\delta}\left(\mathbf{x}_{J}\right)=\operatorname{perm}\left(\left(\frac{1}{\left(x_{i}+x_{j}\right)^{\delta}}\right)_{i, j \in J}\right) .
$$

Lemma 3.6. [1] Let $A(J)=\left(a_{i j}\right)_{i, j \in J}$ be a $m \times m$ matrix and let us assume that $A$ is a most of rank 2 and that all the coefficients $a_{i j}$ are non-zero. Then

$$
\operatorname{det}\left(\left(a_{i j}^{-2}\right)_{i, j \in J}\right)=\operatorname{det}\left(\left(a_{i j}^{-1}\right)_{i, j \in J}\right) \operatorname{perm}\left(\left(a_{i j}^{-1}\right)_{i, j \in J}\right)
$$

Since the $m \times m$ matrix $A(J)=\left(\left(x_{i}+x_{j}\right)_{i, j \in J}\right)$ is indeed of rank 2 (because the range of $\left.A(J)=\operatorname{span}\left(\mathbf{1}, \mathbf{x}_{J}\right)\right)$ and all its coefficients are non-zero, we obtain as a corollary of the above lemma that

$$
D_{2}\left(\mathbf{x}_{J}\right)=D_{1}\left(\mathbf{x}_{J}\right) P_{1}\left(\mathbf{x}_{J}\right) .
$$

Note that this equality is also known as Borchardt's identity.

As a consequence, we obtain using lemma 3.5 that

$$
D_{\delta}\left(\mathbf{x}_{J}\right) \leq \frac{1}{\Gamma(\delta)^{m}} P_{1}\left(\mathbf{x}_{J}\right)^{\delta-1} D_{1}\left(\mathbf{x}_{J}\right) .
$$

By the definition of the permanent, we moreover have

$$
P_{1}\left(\mathbf{x}_{J}\right)=\sum_{\sigma \in \mathcal{S}(J)} \prod_{i \in J} \frac{1}{x_{i}+x_{\sigma(i)}} \leq \sum_{\sigma \in \mathcal{S}(J)} \prod_{i \in J} \frac{1}{x_{i}} \leq m ! \prod_{i=1}^{m} \frac{1}{x_{i}},
$$

since the $x_{i}$ are ordered. Now, since $x_{i} \geq(i-1) \frac{d}{2}$ and $x_{1} \geq \frac{d}{2}$, we obtain

$$
P_{1}\left(\mathbf{x}_{J}\right) \leq m !\left(\frac{2}{d}\right)^{m}\left(\prod_{i=1}^{m-1} \frac{1}{i}\right)=m\left(\frac{2}{d}\right)^{m} .
$$


We also know that for $1 \leq \delta \leq 2, \Gamma(\delta) \geq \frac{1}{2}$, so (12) finally implies that

$$
D_{\delta}\left(\mathbf{x}_{J}\right) \leq 2^{m}\left(m\left(\frac{2}{d}\right)^{m}\right)^{\delta-1} D_{1}\left(\mathbf{x}_{J}\right) \leq m\left(\frac{4}{d}\right)^{m} D_{1}\left(\mathbf{x}_{J}\right)
$$

and this completes the first step of the proof.

\section{Proof of theorem 3.1: second step.}

For notational simplicity, we rewrite the vector $\mathbf{x}_{J}$ as $\mathbf{x}_{J}=\frac{d}{2} \mathbf{z}$, where $\mathbf{z}=\left(z_{1}, \ldots, z_{m}\right)$ and $z_{1} \leq \ldots \leq z_{m}$. We therefore have

$$
D_{1}\left(\mathbf{x}_{J}\right)=\left(\frac{2}{d}\right)^{m} D_{1}(\mathbf{z})
$$

Because of the constraint on $x_{1}$, we obtain that $z_{1} \geq 1$. We are looking for a uniform upper bound on $D_{1}(\mathbf{z})$ over all vectors $\mathbf{z} \in \mathcal{Z}=\left\{\mathbf{z} \in\left[1, \infty\left[{ }^{m}: z_{1} \leq \ldots \leq z_{m}\right\}\right.\right.$, so we may as well assume that $z_{1}=1$; indeed, if $z_{1}>1$, then $\mathbf{z}^{\prime}=\frac{1}{z_{1}} \mathbf{z}$ is such that $z_{1}^{\prime}=1$ and

$$
D_{1}(\mathbf{z})=\left(\frac{1}{z_{1}}\right)^{m} D_{1}\left(\mathbf{z}^{\prime}\right)<D_{1}\left(\mathbf{z}^{\prime}\right)
$$

Notice that

$$
D_{1}(\mathbf{z})=\left(\prod_{1 \leq i<j \leq m} \frac{z_{j}-z_{i}}{z_{i}+z_{j}}\right)^{2} \prod_{1 \leq i \leq m} \frac{1}{2 z_{i}} \leq \prod_{1 \leq i \leq m} \frac{1}{2 z_{i}},
$$

so $D_{1}$ reaches its supremum on the set $\mathcal{Z}$. We define next the function $V_{1}$ by

$$
V_{1}(\mathbf{z})=-\log D_{1}(\mathbf{z})=2 \sum_{1 \leq i<j \leq m} \log \left(\frac{z_{i}+z_{j}}{z_{j}-z_{i}}\right)+\sum_{1 \leq i \leq m} \log \left(z_{i}\right)+m \log (2) .
$$

Lemma 3.7. Let $\mathbf{z} \in \mathcal{Z}$ be such that $V_{1}(\mathbf{z})$ reaches its infimum at $\mathbf{z}$. If $1 \leq p \leq k<l \leq m$, then

$$
(k-p+1)(l-k) \frac{z_{p} z_{l}}{z_{l}^{2}-z_{p}^{2}} \leq \frac{m-k}{4} .
$$

Proof. If $g(t)=V_{1}\left(z_{1}, \ldots, z_{k}, t z_{k+1}, \ldots, t z_{m}\right)$, then

$$
g^{\prime}(t)=2 \sum_{\substack{1 \leq i \leq k \\ k<j \leq m}}\left[\log \left(\frac{t z_{j}+z_{i}}{t z_{j}-z_{i}}\right)\right]^{\prime}+\frac{m-k}{t}=2 \sum_{\substack{1 \leq i \leq k \\ k<j \leq n}}\left(\frac{z_{j}}{t z_{j}+z_{i}}-\frac{z_{j}}{t z_{j}-z_{i}}\right)+\frac{m-k}{t},
$$

so

$$
g^{\prime}(1)=-4 \sum_{\substack{1 \leq i \leq k \\ k<j \leq m}} \frac{z_{i} z_{j}}{z_{j}^{2}-z_{i}^{2}}+m-k
$$

Since $V_{1}$ is minimum in $\mathbf{z}$, we have $g^{\prime}(1)=0$, i.e.

$$
\sum_{\substack{1 \leq i \leq k \\ k<j \leq m}} \frac{z_{i} z_{j}}{z_{j}^{2}-z_{i}^{2}}=\frac{m-k}{4} .
$$

But $\frac{z_{j} z_{i}}{z_{j}^{2}-z_{i}^{2}}=\frac{1}{\frac{z_{j}}{z_{i}}-\frac{z_{i}}{z_{j}}}$ increases if $i$ increases or $j$ decreases, so

$$
\frac{z_{p} z_{l}}{z_{l}^{2}-z_{p}^{2}} \leq \frac{z_{i} z_{j}}{z_{j}^{2}-z_{i}^{2}}, \quad \text { whenever } \quad p \leq i \leq k \quad \text { and } \quad k<j \leq l .
$$


Therefore,

$$
\frac{m-k}{4} \geq \sum_{\substack{p \leq i \leq k \\ k<j \leq l}} \frac{z_{i} z_{j}}{z_{j}^{2}-z_{i}^{2}} \geq(k-p+1)(l-k) \frac{z_{p} z_{l}}{z_{l}^{2}-z_{p}^{2}}
$$

Corollary 3.8. Let $\mathbf{z} \in \mathcal{Z}$ be such that $V_{1}(\mathbf{z})$ reaches its infimum at $\mathbf{z}$. If $1 \leq p \leq k<l \leq m$, then

$$
\frac{z_{l}}{z_{p}} \geq \frac{4(k-p+1)(l-k)}{m-k}
$$

Proof. Let $x>0$ and $\beta>0$. If $x-\frac{1}{x} \geq 2 \beta$, then $x \geq \beta+\sqrt{1+\beta^{2}} \geq 2 \beta$. The corollary follows by combining this fact with inequality (15) (taking $x=\frac{z_{l}}{z_{p}}$ and $\beta=\frac{2(k-p+1)(l-k)}{m-k}$ ).

The proof of theorem 3.1 relies on the following key estimate.

Lemma 3.9. There exists a constant $K>0$ independent of $m$ such that

$$
V_{1}(\mathbf{z}) \geq K m^{3 / 2}, \quad \text { or equivalently, } \quad D_{1}(\mathbf{z}) \leq \exp \left(-K m^{3 / 2}\right),
$$

for all $\mathbf{z} \in \mathcal{Z}$.

Note that there exists a (sort of) converse to the above lemma; see the appendix.

Proof. Let $\mathbf{z} \in \mathcal{Z}$ be the point where $V_{1}$ reaches its infimum. Let $r=1+\lfloor\sqrt{m-1}\rfloor$, where $\lfloor\sqrt{m-1}\rfloor$ is the integer part of $\sqrt{m-1}$. By (17), we have

$$
\frac{z_{k+r}}{z_{k-r+1}} \geq \frac{4 r^{2}}{m-k} \geq \frac{4 r^{2}}{m-1} \geq 4, \quad \text { if } \quad r \leq k \leq m-r
$$

i.e.

$$
\frac{z_{s+2 r-1}}{z_{s}} \geq 4, \quad \text { if } \quad 1 \leq s \leq m+1-2 r, \quad \text { so } \quad z_{t(2 r-1)+1} \geq 4^{t} .
$$

Let us write $m=(2 r-1) t+q(q$ and $t$ integers, $0 \leq q<2 r-1)$. We obtain that

$$
\begin{aligned}
\sum_{1 \leq i \leq m} \log z_{i} & \geq(2 r-1) \log 1+(2 r-1) \log 4+\cdots+(2 r-1) \log \left(4^{t-1}\right)+q \log \left(4^{t}\right) \\
& \geq(2 r-1) \frac{t(t-1)}{2} \log 4 .
\end{aligned}
$$

So for all $\varepsilon>0$, there exists $m_{0} \geq 1$ such that

$$
\sum_{1 \leq i \leq m} \log z_{i} \geq m^{3 / 2}(1-\epsilon) \frac{\log 4}{4}, \quad \text { whenever } m \geq m_{0} .
$$

This concludes the proof, since $V_{1}(\mathbf{z}) \geq \sum_{1 \leq i \leq m} \log z_{i}+m \log (2)$.

\section{Proof of theorem 3.1: conclusion.}

Let us now gather together all estimates. By (5) and (6), we have

$$
\begin{aligned}
\exp \left(C_{n} / 2\right) & \leq \operatorname{det}\left(I+\sqrt{n P} G^{(\delta)}\right)=\sum_{J \subset\{1, \ldots, n\}} \operatorname{det}\left(\sqrt{n P} G^{(\delta)}(J)\right) \\
& =\sum_{J \subset\{1, \ldots, n\}}(n P)^{|J| / 2} D_{\delta}\left(\mathbf{x}_{J}\right)=\sum_{m=0}^{n}(n P)^{m / 2} \sum_{J \subset\{1, \ldots, n\}:|J|=m} D_{\delta}\left(\mathbf{x}_{J}\right) .
\end{aligned}
$$


Using (13), (14) and (18), we obtain successively that

$$
\begin{aligned}
\exp \left(C_{n} / 2\right) & \leq \sum_{m=0}^{n}(n P)^{m / 2} m\left(\frac{4}{d}\right)^{m} \sum_{J \subset\{1, \ldots, n\}:|J|=m} D_{1}\left(\mathbf{x}_{J}\right) \\
& \leq \sum_{m=0}^{n}(n P)^{m / 2} m\left(\frac{4}{d}\right)^{m} \sum_{J \subset\{1, \ldots, n\}:|J|=m}\left(\frac{2}{d}\right)^{m} \exp \left(-K m^{3 / 2}\right) .
\end{aligned}
$$

Since

$$
\sum_{J \subset\{1, \ldots, n\}:|J|=m} 1=\left(\begin{array}{c}
n \\
m
\end{array}\right) \leq n^{m},
$$

we have

$$
\exp \left(C_{n} / 2\right) \leq \sum_{m=0}^{n} P^{m / 2} n^{3 m / 2} m\left(\frac{8}{d^{2}}\right)^{m} \exp \left(-K m^{3 / 2}\right) \leq \sum_{m=0}^{n} \exp \left(L m \log n-K m^{3 / 2}\right),
$$

where $L$ is some positive constant. Choosing $m_{0}=\left(\frac{L \log n}{K}\right)^{2}$, we moreover have

$$
\begin{aligned}
\exp \left(C_{n} / 2\right) & \leq \sum_{m=0}^{m_{0}-1} \exp \left(L m \log (n)-K m^{3 / 2}\right)+\sum_{m=m_{0}}^{n} \exp \left(L m \log n-K m^{3 / 2}\right) \\
& \leq \sum_{m=0}^{m_{0}-1} \exp \left(L m_{0} \log n\right)+\sum_{m=m_{0}}^{n} 1=m_{0} \exp \left(L m_{0} \log n\right)+n
\end{aligned}
$$

Since $m_{0}=\left(\frac{L \log n}{K}\right)^{2}$, we conclude that for all $\varepsilon>0$, there exists $M>0$ sufficiently large such that

$$
\exp \left(C_{n} / 2\right) \leq \exp \left(M(\log n)^{3+\varepsilon}\right),
$$

which concludes the proof of theorem 3.1.

\section{Acknowledgement}

We would like to thank Alexei Borodin, Emre Telatar and Ofer Zeitouni for stimulating discussions.

\section{A Appendix}

In this appendix, we show that the order of magnitude obtained in (18) is not only an upper bound, but is also tight.

Proposition A.1. There exists a constant $K>0$ independent of $m$ such that

$$
V_{1}(\mathbf{z}) \leq K m^{3 / 2}, \quad \text { if } z_{j}=\exp \left(\frac{j-1}{\sqrt{m}}\right), j=1, \ldots, m .
$$

Proof. It is clearly sufficient to prove the above relation for $m=r^{2}$ with $r$ integer. Since

$$
\sum_{1 \leq i \leq m} \log \left(z_{i}\right)=\sum_{1 \leq i \leq m} \frac{i-1}{\sqrt{m}}=\frac{m(m-1)}{2 \sqrt{m}}=O\left(m^{3 / 2}\right)
$$


we only need to prove that

$$
\sum_{1 \leq i<j \leq m} \log \left(\frac{z_{i}+z_{j}}{z_{j}-z_{i}}\right)=\sum_{1 \leq l \leq m-1}(m-l) \log \left(\frac{1+e^{-l / r}}{1-e^{-l / r}}\right) \quad \text { is } \quad O\left(m^{3 / 2}\right) .
$$

Let now

$$
s=\sum_{1 \leq l \leq r}(m-l) \log \left(\frac{1+e^{-l / r}}{1-e^{-l / r}}\right) \quad \text { and } \quad t=\sum_{r+1 \leq l \leq m-1}(m-l) \log \left(\frac{1+e^{-l / r}}{1-e^{-l / r}}\right) .
$$

Since

$$
\log \left(\frac{1+y}{1-y}\right)=2 \sum_{k \text { odd }} \frac{y^{k}}{k} \leq 2 \sum_{k \text { odd }} y^{k}=\frac{2 y}{1-y^{2}}, \quad \text { for } 0<y<1,
$$

we obtain that

$$
t \leq 2 m \sum_{r+1 \leq l \leq m-1} \frac{e^{-l / r}}{1-e^{-2 l / r}} \leq \frac{2 m}{1-e^{-2}} \sum_{l=r+1}^{\infty} e^{-l / r} \leq \frac{2 m}{1-e^{-2}} \frac{e^{-1}}{1-e^{-1 / r}} .
$$

But $1-e^{-1 / r}=\frac{1}{r} e^{-c}$ for some $0<c<\frac{1}{r}$, so

$$
t \leq \frac{2 m}{1-e^{-2}} e^{-1} r e^{c} \leq \frac{2 r m}{1-e^{-2}},
$$

which is $O\left(m^{3 / 2}\right)$. On the other hand, if $u>0$, we have $1-e^{-u}=u e^{-c}$ with $0<c<u$, hence $\log \left(1-e^{-u}\right)=\log u-c$. Therefore, for $1 \leq l \leq r,-\log \left(1-e^{-l / r}\right) \leq \log \left(\frac{r}{l}\right)+1$ and

$$
s \leq m \sum_{1 \leq l \leq r}\left(\log 2+\log \left(\frac{r}{l}\right)+1\right)=m r(1+\log 2)+m \log \left(\frac{r^{r}}{r !}\right),
$$

and since $\log \left(\frac{r^{r}}{r !}\right) \sim r, s$ is also $O\left(m^{3 / 2}\right)$.

\section{References}

[1] Carlitz, L.; Levine, J. An Identity of Cayley. Amer. Math. Monthly 67 (6), 1960, 571-573.

[2] Cover, T. M.; Thomas, J. A. Elements of Information Theory. John Wiley \& Sons, 1991, New York.

[3] Gupta, P.; Kumar, P. R. The Capacity of Wireless Networks. IEEE Trans. on Information Theory 42 (2), 2000, 388-404.

[4] Jovicic, A.; Kulkarni, S. R.; Viswanath, P. Upper Bounds to Transport Capacity of Wireless Networks. IEEE Trans. on Information Theory 50 (11), 2004, 2555-2565.

[5] Kumar, P. R.; Xie, L.-L. A Network Information Theory for Wireless Communications: Scaling Laws and Optimal Operation. IEEE Trans. on Information Theory 50 (5), 2004, 748-767.

[6] Lévêque, O.; Telatar, E. Information Theoretic Upper Bounds on the Capacity of Large Extended Ad Hoc Wireless Networks. To appear in the IEEE Trans. on Information Theory, March 2005.

[7] Weyl, H. The Classical Groups. Their Invariants and Representations. Princeton University Press, Princeton, N.J., 1939. 